\title{
Plasma and wave phenomena induced by neutral gas releases in the solar wind
}

\author{
H. Laakso ${ }^{1}$, R. Grard ${ }^{1}$, P. Janhunen ${ }^{2}$, and J.-G. Trotignon ${ }^{3}$ \\ ${ }^{1}$ ESA Space Science Department, Noordwijk, The Netherlands \\ ${ }^{2}$ Finnish Meteorological Institute, Geophysics Research, Helsinki, Finland \\ ${ }^{3}$ LPCE, CNRS, Orleans, France
}

Received: 19 April 2000 - Revised: 20 July 2001 - Accepted: 23 August 2001

\begin{abstract}
We investigate plasma and wave disturbances generated by nitrogen $\left(\mathrm{N}_{2}\right)$ gas releases from the cooling system of an IR-camera on board the Vega 1 and Vega 2 spacecraft, during their flybys of comet Halley in March 1986. $\mathrm{N}_{2}$ molecules are ionized by solar UV radiation at a rate of $\sim 7 \cdot 10^{-7} \mathrm{~s}^{-1}$ and give rise to a plasma cloud expanding around the spacecraft. Strong disturbances due to the interaction of the solar wind with the $\mathrm{N}_{2}^{+}$ion cloud are observed with a plasma and wave experiment (APV-V instrument). Three gas releases are accompanied by increases in cold electron density and simultaneous decreases of the spacecraft potential; this study shows that the spacecraft potential can be monitored with a reference sensor mounted on a short boom. The comparison between the model and observations suggests that the gas expands as an exhaust plume, and approximately only $1 \%$ of the ions can escape the beam within the first meters. The releases are also associated with significant increases in wave electric field emission $(8 \mathrm{~Hz}-$ $300 \mathrm{kHz}$ ); this phenomenon lasts for more than one hour after the end of the release, which is most likely due to the temporary contamination of the spacecraft surface by nitrogen gas. DC electric fields associated with the events are complex but interesting. No magnetic field perturbations are detected, suggesting that no significant diamagnetic effect (i.e. magnetic cavity) is associated with these events.
\end{abstract}

Key words. Ionosphere (planetary ionosphere) - Space plasma physics (active perturbation experiments; instruments and techniques)

\section{Introduction}

Gases are commonly expelled from spacecraft for various applications, such as orbit and attitude control, or instrument/detector cooling (Burke, 1983). Volatile chemicals have also been released in the solar wind in order to investigate the interaction processes between the solar wind and

Correspondence to: H. Laakso (hlaakso@so.estec.esa.nl) gas clouds (e.g. Valenzuela et al., 1986). The dynamics of the Earth's ionosphere and magnetosphere has been studied by means of gas releases (e.g. Barium, Lithium, Nitrogen, Argon, etc.) expanding around rockets and satellites (e.g. Holmgren et al., 1980) and around the Space Shuttle (e.g. Sasaki, 1988). In all cases, photoionization of neutral particles creates an expanding plasma cloud which results in plasma and wave disturbances. When the leakage rate is high and the ionization time is short, a magnetic cavity forms (Lühr et al., 1986) and the wave activity within the cavity ceases (Gurnett et al., 1986b; Koons and Anderson, 1988).

This investigation deals with disturbances generated by nitrogen releases made for cooling the infrared spectrometer (IKS) on the Vega spacecraft. The object of IKS is to measure the radiation from the inner coma of comet Halley (Arduine et al., 1983). To improve the sensitivity of the instrument, the detectors are cooled down to $77 \mathrm{~K}$ by the Joule-Thomson expansion of a gas. For that purpose, 700 $\mathrm{g}$ of nitrogen are stored in four tanks at a pressure of 350 atm. On Vega 1, all tanks are opened at the same time, whereas on Vega 2, two pairs are opened with an interval of $35 \mathrm{~min}$. In spite of the low photoionization rate of $\mathrm{N}_{2}$ molecules $\left(\sim 7 \cdot 10^{-7} \mathrm{~s}^{-1}\right)$, significant perturbations are detected with the plasma and wave experiment APV-V.

This paper reports on the analysis of the Vega release events. Section 2 describes the APV-V experiment and gives some detail about the IKS nitrogen releases. In Sect. 3, we analyze the plasma and electric field phenomena observed with the APV-V sensors. Section 4 summarizes the major findings of this paper.

\section{Instrumentation}

Figure 1 shows the locations of the APV-V sensors (Grard et al., 1989) and the IKS IR-camera (Arduine et al., 1983) on the Vega spacecraft. The quasistatic (dc) and wave (ac) electric fields are measured with a double probe antenna, made of two solid spheres (sensors P1 and P2), $10 \mathrm{~cm}$ in diameter, 


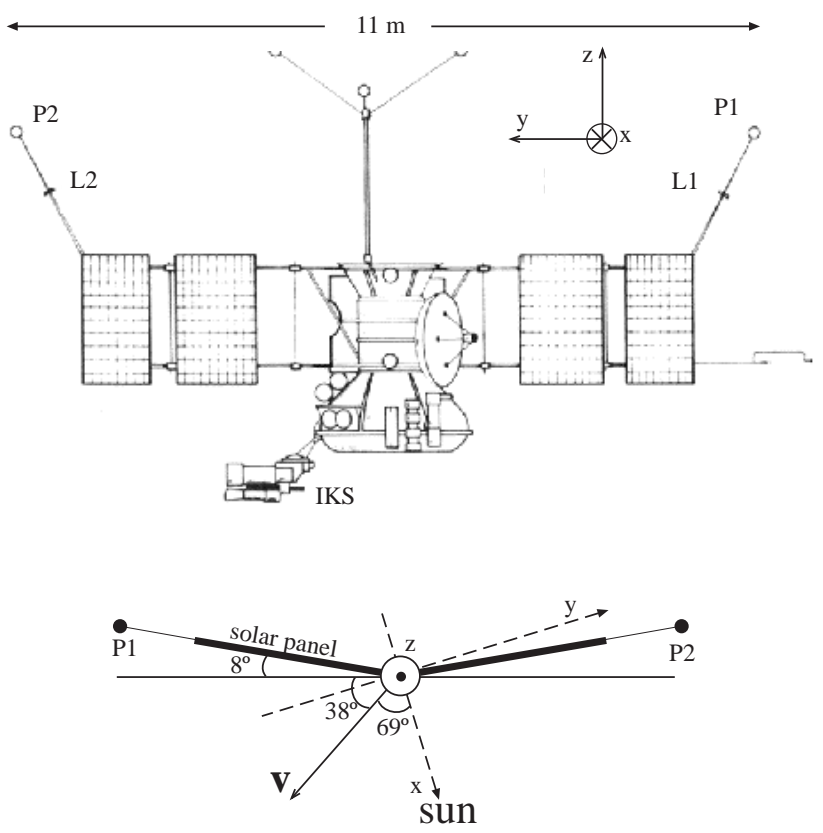

Fig. 1. Top: Outline of the Vega spacecraft showing the locations of the APV-V sensors and IKS instrument. Bottom: Orientation of the satellite structure relative to its velocity vector and the Sun (top view).

located at the tips of two 2-meter long booms and separated by a distance $L=11 \mathrm{~m}$. The potential differences between the probes, $V_{12}=V_{1}-V_{2}$, and between probe $\mathrm{P} 2$ and the spacecraft structure, $V_{2 s}=V_{2}-V_{s}$, are sampled at intervals of $1 \mathrm{~s}$ and $4 \mathrm{~s}$, respectively. The $V_{12}$ voltage difference is also analyzed with a group of 16 adjacent and logarithmically spaced filters in the frequency range of $8 \mathrm{~Hz}-300 \mathrm{kHz}$. The sampling interval is $1 \mathrm{~s}$ for the first four filters (8-14 $\mathrm{Hz}, 14-25 \mathrm{~Hz}, 25-40 \mathrm{~Hz}, 40-75 \mathrm{~Hz}$ ) and $0.5 \mathrm{~s}$ for the twelve other filters $(75-150 \mathrm{~Hz}, 150-300 \mathrm{~Hz}, 300-600 \mathrm{~Hz}, 0.6-1.2$ $\mathrm{kHz}, 1.2-2.4 \mathrm{kHz}, 2.4-4.8 \mathrm{kHz}, 4.8-9.6 \mathrm{kHz}, 9.6-19 \mathrm{kHz}$, $19-38 \mathrm{kHz}, 38-76 \mathrm{kHz}, 76-150 \mathrm{kHz}, 150-300 \mathrm{kHz})$.

The Langmuir probes (L1 and L2) are mounted at mid length along the booms; they are cylindrical and have a collecting area $A=4.4 \mathrm{~cm}^{2}$. Probe $\mathrm{L} 2$ is biased at a fixed potential of $+5 \mathrm{~V}$ with respect to the spacecraft structure in order to detect fast fluctuations of the electron flux. The current response of probe $\mathrm{L} 1$ is measured while its potential is swept with a period of $32 \mathrm{~s}$ between $-6 \mathrm{~V}$ and $+6 \mathrm{~V}$ on Vega 1 and between $-4 \mathrm{~V}$ and $+2 \mathrm{~V}$ on Vega 2 (Grard et al., 1989).

The infrared spectrometer IKS (Fig. 1) is cooled down by releasing low temperature $(77 \mathrm{~K})$ nitrogen molecules $\left(\mathrm{N}_{2}\right)$ during the approach in the cometosheath, at distances of $(7-6) \cdot 10^{5} \mathrm{~km}$ from the nucleus (Fig. 2), where the solar wind speed is approximately $500 \mathrm{~km} \mathrm{~s}^{-1}$ (Gringauz et al., 1986), and the plasma density is of the order of several tens of electrons per $\mathrm{cm}^{3}$ (Grard et al., 1989; Laakso, 1990). The gas is expanding through a nozzle in the $-z$ direction, with a velocity $\mathrm{v}_{n} \sim 200 \mathrm{~m} \mathrm{~s}^{-1}$; the $\mathrm{N}_{2}$ release rates are plotted against cometocentric distance in Fig. 3: $K\left(\mathrm{~g} \mathrm{~min}^{-1}\right)$

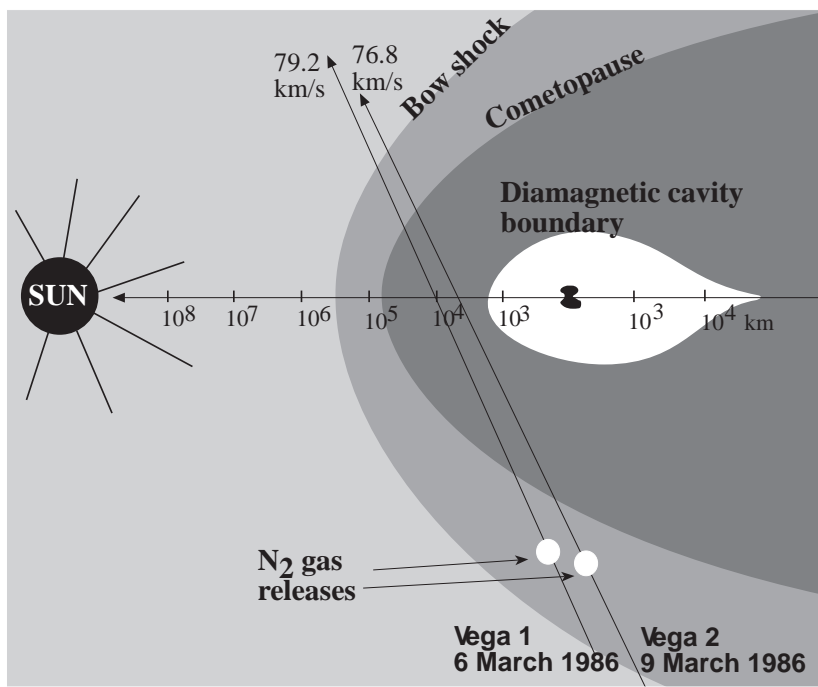

Fig. 2. Locations of the $\mathrm{N}_{2}$ gas releases along the Vega 1 and Vega 2 trajectories during their comet Halley approaches.
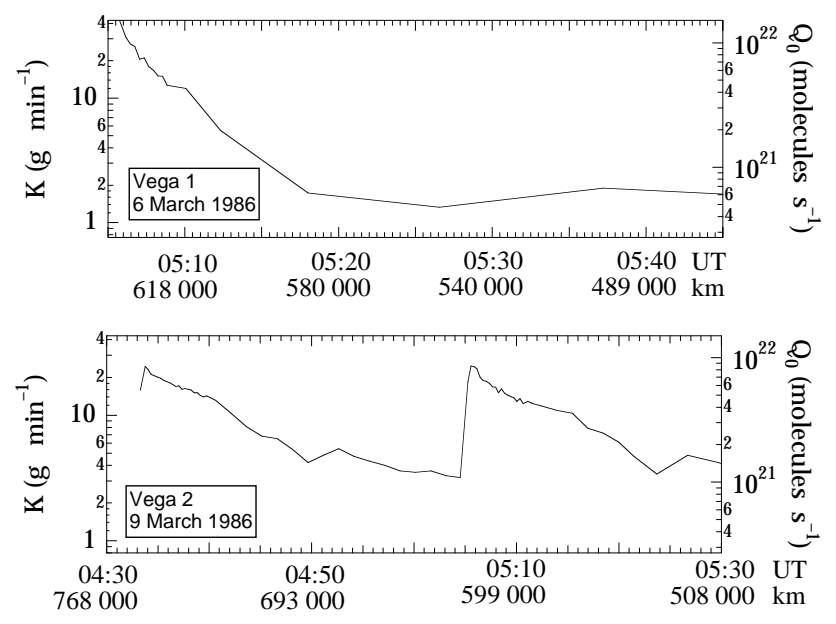

Fig. 3. Nitrogen gas release rates on Vega 1 and 2.

is given along the left-hand axis and $Q_{0}$ (molecules s ${ }^{-1}$ ) is given along the right-hand axis. The two numbers are related by the expression

$Q_{0}=\frac{N_{A}}{m_{0}} \frac{K}{60}=3.6 \cdot 10^{20} \mathrm{~K}$,

where $N_{A}=6.022 \cdot 10^{23}$ molecules $/ \mathrm{mol}$ is the Avogadro's number and $m_{0}=28.02 \mathrm{~g} / \mathrm{mol}$ is the molar mass of $\mathrm{N}_{2}$. The start times of the releases are 05:05:30 UT for Vega 1 and 04:33:00 UT and 05:05:06 UT for Vega 2.

The spacecraft attitude is 3-axis stabilized during the flybys and the coordinate system is derived from the cometocentric solar ecliptic system by a rotation $\alpha$ around the common $\mathrm{z}$ axis where $\alpha$ is $18^{\circ}$ for Vega 1 and $15^{\circ}$ for Vega 2 . 

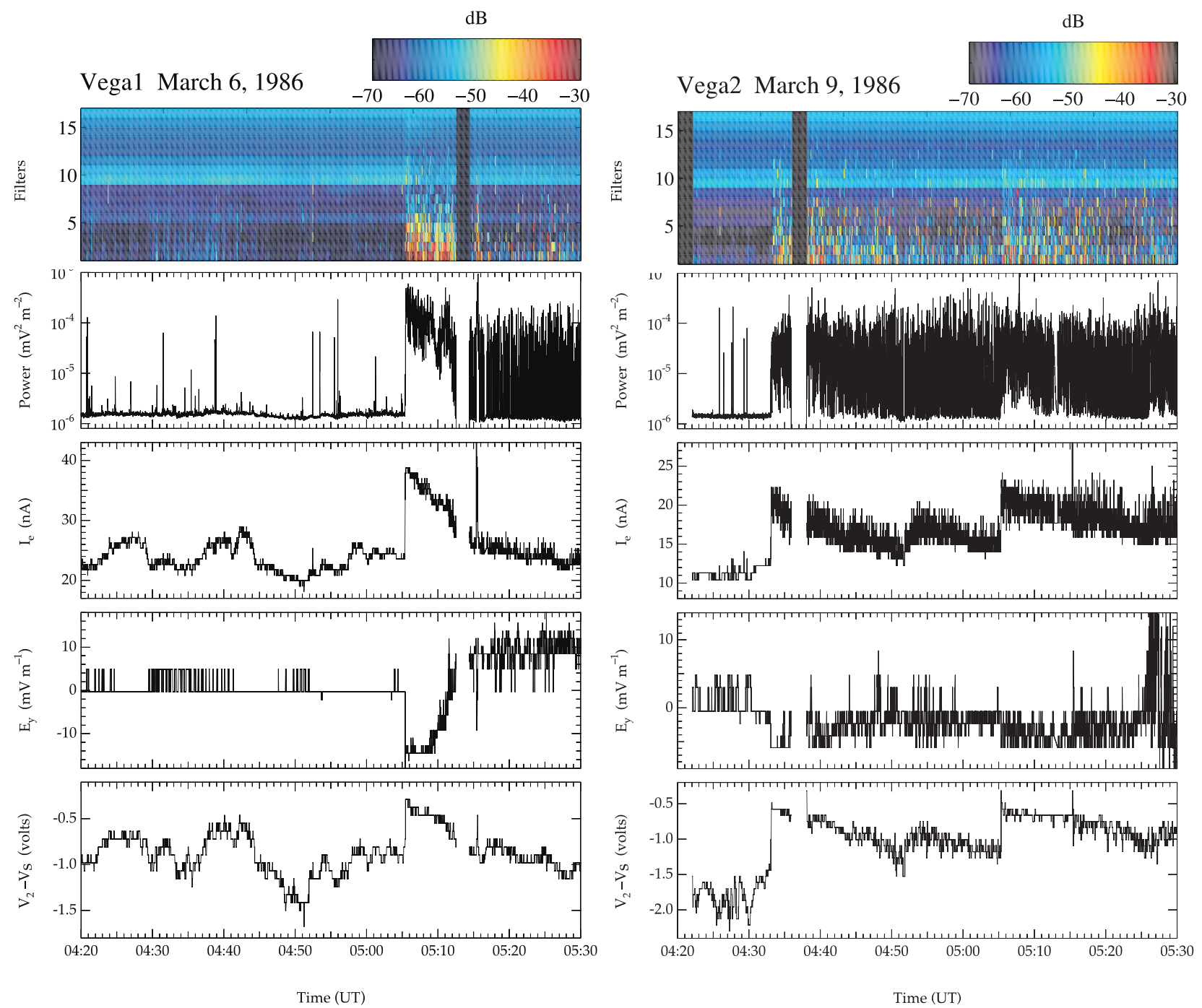

Fig. 4. Summary plots of the plasma and wave disturbances observed during the gas releases on (a) Vega 1 and (b) Vega 2 (see text for details).

\section{Measurements}

Each $\mathrm{N}_{2}$ release is accompanied by simultaneous enhancements of electron flux and wave electric fields, as shown in Figs. 4a and $4 \mathrm{~b}$ for Vega 1 and Vega 2, respectively. The quantities represented in the five panels are, from top to bottom, signals measured with the electric antenna in 16 adjacent filters covering the frequency range of $8 \mathrm{~Hz}-300 \mathrm{kHz}$ $(0 \mathrm{~dB}$ corresponds to $1 \mathrm{~V}$ r.m.s.), the square of the electric field integrated over the whole frequency range, the electron current collected by L2, the equivalent dc electric field $\left(V_{12} / L\right)$, and the potential difference $V_{2 s}$ between probe P2 and the spacecraft. Data gaps correspond to calibration intervals of the APV-V instrument. The ionization rate $\alpha$ of $\mathrm{N}_{2}$ at $1 \mathrm{AU}$ is $(4.9 \pm 2.3) \cdot 10^{-7} \mathrm{~s}^{-1}$ (Banks and Kockarts, 1973), i.e. $(7.8 \pm 3.7) \cdot 10^{-7} \mathrm{~s}^{-1}$ for Vega 1 (at $0.79 \mathrm{AU}$ ) and $(7.0 \pm 3.3) \cdot 10^{-7} \mathrm{~s}^{-1}$ for Vega 2 (at $0.83 \mathrm{AU}$ ).

\subsection{Electron flux}

Figure 5 displays the electron current shown in the third panels of Figs. $4 a$ and $4 b$, against the release rate K. The best fit to the data points collected during the first Vega 2 release (squares) is given by

$I_{e}=0.33 K+13$,

where $I_{e}$ and $K$ are expressed in nA and $\mathrm{g} / \mathrm{min}$, respectively; this empirical relation is represented by a solid line in Fig. 5 . The background current at $K=0$ is approximately $13 \mathrm{nA}$.

The data points taken during the second Vega 2 release (crosses) lie primarily above the solid line, except at the beginning of the event ( $K \approx 25 \mathrm{~g} / \mathrm{min})$. Thereafter, the current variation is approximated by a dotted line, which has the same slope but a different offset, $15.8 \mathrm{nA}$ at $K=0$. This behavior indicates that the ambient electron current is increasing shortly after the onset of the second release, as also evidenced by the middle panel of Fig. $4 \mathrm{~b}$. 


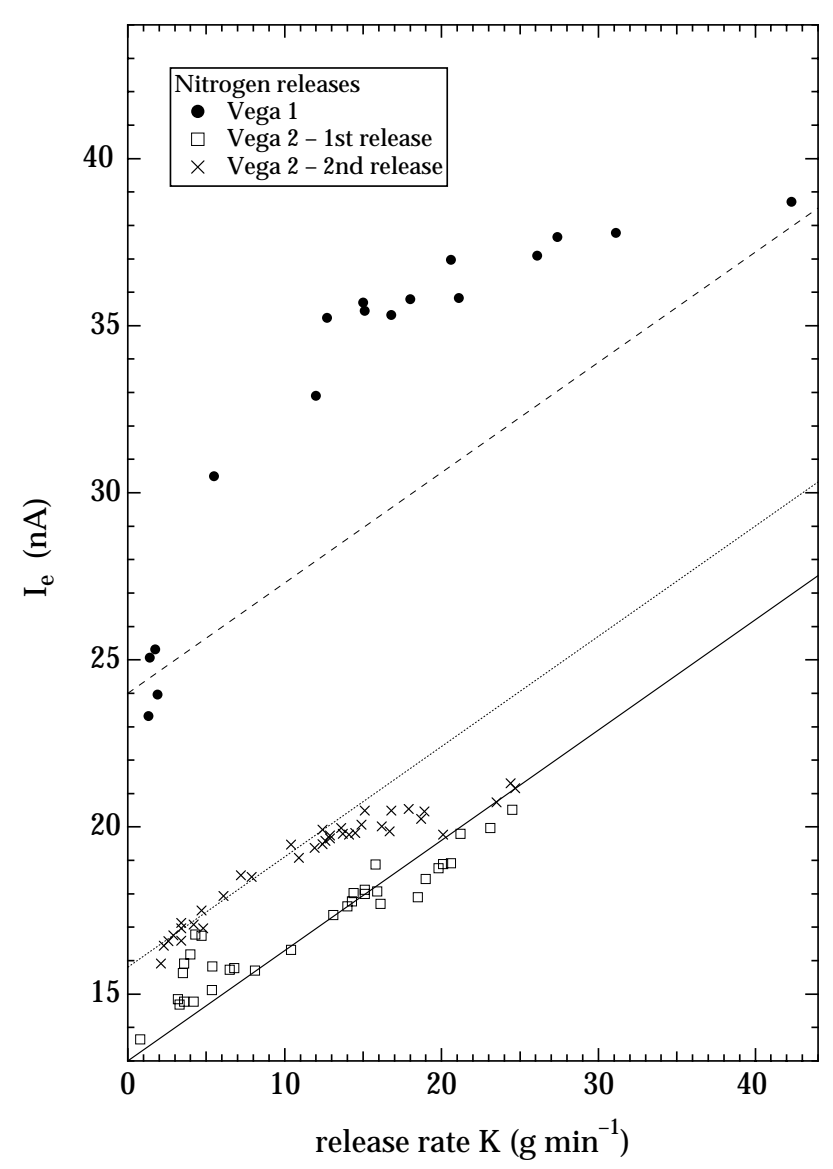

Fig. 5. Electron currents, measured with Langmuir probe L2, against release rate; full circles - Vega 1 release; open squares first Vega 2 release; crosses - second Vega 2 release.

The current observed during the Vega 1 release does not vary linearly with $K$, probably due to the fact that the plasma environment is fluctuating during the event. The dashed line, representing the equation $I_{e}=0.33 K+24$, fits, however, the measurements for large and small values of $K$, i.e. at the beginning and at the end of the release. Then, the ambient electron current is approximately $24 \mathrm{nA}$, as it appears in the middle panel of Fig. 4a. However, most data points lie above the dashed line, since the background current is not constant during the event.

Subtracting the current increment $\Delta I_{e}=0.33 \mathrm{~K}$ associated with the nitrogen release from the measurements yields the ambient electron current shown in Fig. 6. Note that the quasi-periodic fluctuation, observed before 05:05 UT on 6 March 1986 (Fig. 4a), probably continues during the cooling operation.

\subsubsection{Spherical expansion}

We shall now derive analytical expressions of the current increment $\Delta I_{e}$ for two different models of the neutral gas flow. We first assume a spherical expansion with a radial speed $v_{n}$ and an ionization rate $\alpha$. The neutral density at a distance $r$
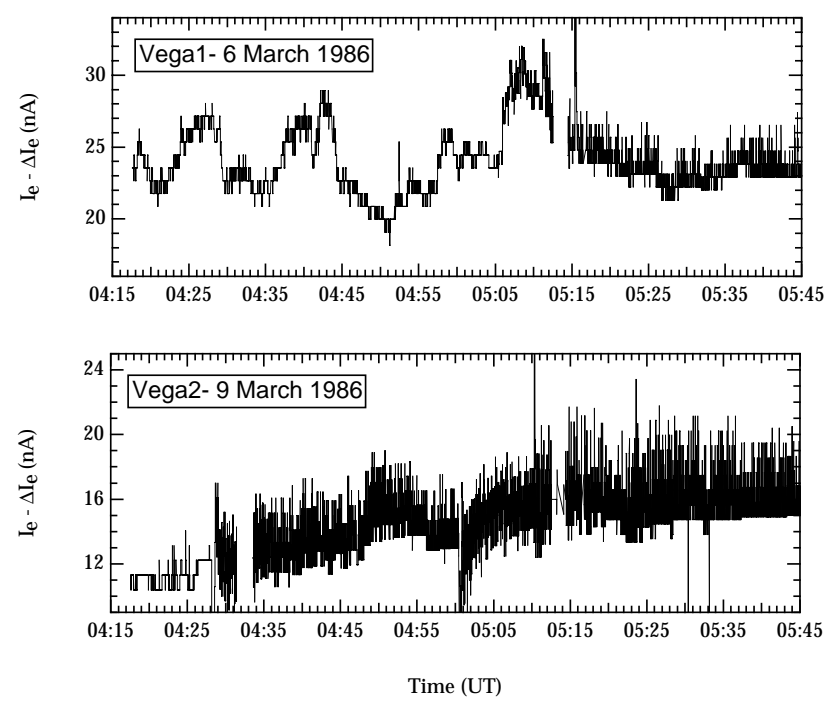

Fig. 6. Ambient electron flux in the outer cometosheath on Vega 1 (top) and Vega 2 (bottom) after correction for the gas release effects.

is given by

$n_{n}=\frac{Q_{0}}{4 \pi v_{n} r^{2}} \exp \left(-\frac{\alpha r}{v_{n}},\right)$

and the nitrogen ion density at the location of the probe is simply

$n_{i}=\frac{Q_{0}}{4 \pi v_{n} r_{0}^{2}}\left[1-\exp \left(-\frac{\alpha r_{0}}{v_{n}}\right)\right] \approx \frac{\alpha Q_{0}}{4 \pi r_{0} v_{n}^{2}}$,

where $r_{0} \sim 8 \mathrm{~m}$ is the distance between the sensor and the source (Fig. 1). Thus, assuming that the ion and electron densities are equal, the current enhancement is

$\Delta I_{e}=n_{i}$ e $v_{e} S=\frac{e \alpha Q_{0} S v_{e}}{4 \pi r_{0} v_{n}^{2}}$,

where $v_{e}$ is the electron thermal velocity, $e$ is the charge of an electron, and $S$ is the collection area of the probe.

The collection area $S$ of a positive probe is approximately $A\left(1+V / V_{e}\right)$ (Grard et al., 1989), where $A \approx 4.4 \mathrm{~cm}^{2}$ is the surface area of the probe, $V$ is the potential of the probe with respect to the plasma, and $V_{e}$ is the electron kinetic energy in volts (i.e. $V_{e}=T_{e} / e$ ). The measurements performed with the Langmuir probe L1 yield a mean kinetic energy of $0.5-1 \mathrm{eV}$, a typical value for photoelectrons (Grard et al., 1989), which implies that no substantial cooling results from electron-neutral collisions. The neutral gas density around the spacecraft varies during the release and lies in the range $10^{17}-10^{18} \mathrm{~m}^{-3}$. The electron-neutral mean free path is then of the order of several meters (Banks and Kockarts, 1973), and, therefore, the electrons collected by the Langmuir probes should indeed not be thermalized by the neutral gas. If $V_{e}=0.5-1 \mathrm{~V}$, Eq. (5) predicts an enhancement $\Delta I_{e} \sim 20 \mathrm{~K}$, i.e. a result 100 times larger than that directly 
derived from observations. Thus, much fewer electrons are collected by the Langmuir probe than expected on the basis of a spherical expansion.

\subsubsection{Collimated beam}

We may consider, alternatively, that the gas is collimated by the nozzle in the $-z$ direction and expands in a narrow conical beam with a solid angle $\Omega \ll 1$ (Fig. 7). The neutral density at a distance $z$ from the source inside the cone is approximately

$n_{n}=\frac{Q_{0}}{\Omega v_{n} z^{2}}$,

where it is assumed that $\exp \left(-\alpha z / v_{n}\right)=1$. Ions are produced within the neutral beam at the rate

$d q_{i}=\alpha n_{n} d V$,

where the elementary volume is given by $d V=\Omega z^{2} d z$. Let us assume that a fraction $\chi$ of ions is isotropically scattered out from this elementary volume, with a velocity identical to that of the neutrals. Then, the associated ion density outside the beam at the detector is

$d n_{i}=\frac{\chi}{4 \pi R^{2} v_{n}} d q_{i}$

where

$R=\sqrt{\left(z+r_{0} \cos \varphi\right)^{2}+\left(r_{0} \sin \varphi\right)^{2}}$

is the distance of the sensor from the beam point with $r_{0} \approx 8$ $\mathrm{m}$ and $\varphi \approx 45^{\circ}$, as defined in Figure 7 (for the angle, see Figure 1).

Combining Eqs. (6)-(9) and integrating expression (8) with respect to $z$ between 0 and $-\infty$ yields the total nitrogen ion density at the location of probe $\mathrm{L} 1$

$n_{i}=\int_{0}^{\infty} d n_{i}=\frac{\alpha Q_{0}}{4 \pi r_{0} v_{n}^{2}} \times \frac{\varphi}{\cos \varphi}$,

for $\varphi \approx 45^{\circ}, \varphi / \cos \varphi \approx 1.1$. Equations (4) and (10) have similar forms, but it is seen that, contrary to the spherical model, the collimated model agrees with the observations if we assume that $\chi \approx 0.01$. In other words, about $1 \%$ of the $\mathrm{N}_{2}^{+}$ions escape from the beam, which confirms that the nitrogen cloud does not expand spherically and quantifies the efficiency of the nozzle.

\subsection{Spacecraft potential}

The bottom two panels in Figs. $4 \mathrm{a}$ and $4 \mathrm{~b}$ show the potential difference between probe $\mathrm{P} 2$ and the spacecraft, $V_{2 s}=$ $V_{2}-V_{s}$. This quantity is negative because the reference probe is biased with respect to the spacecraft by a constant positive current of $50 \mathrm{nA}$, and thus, the probe assumes a positive potential close to that of the ambient plasma; $V_{2}$ is typically 1-2 volts positive with respect to the ambient plasma. In a rarefied environment, $V_{2}$ is relatively stable because the bias

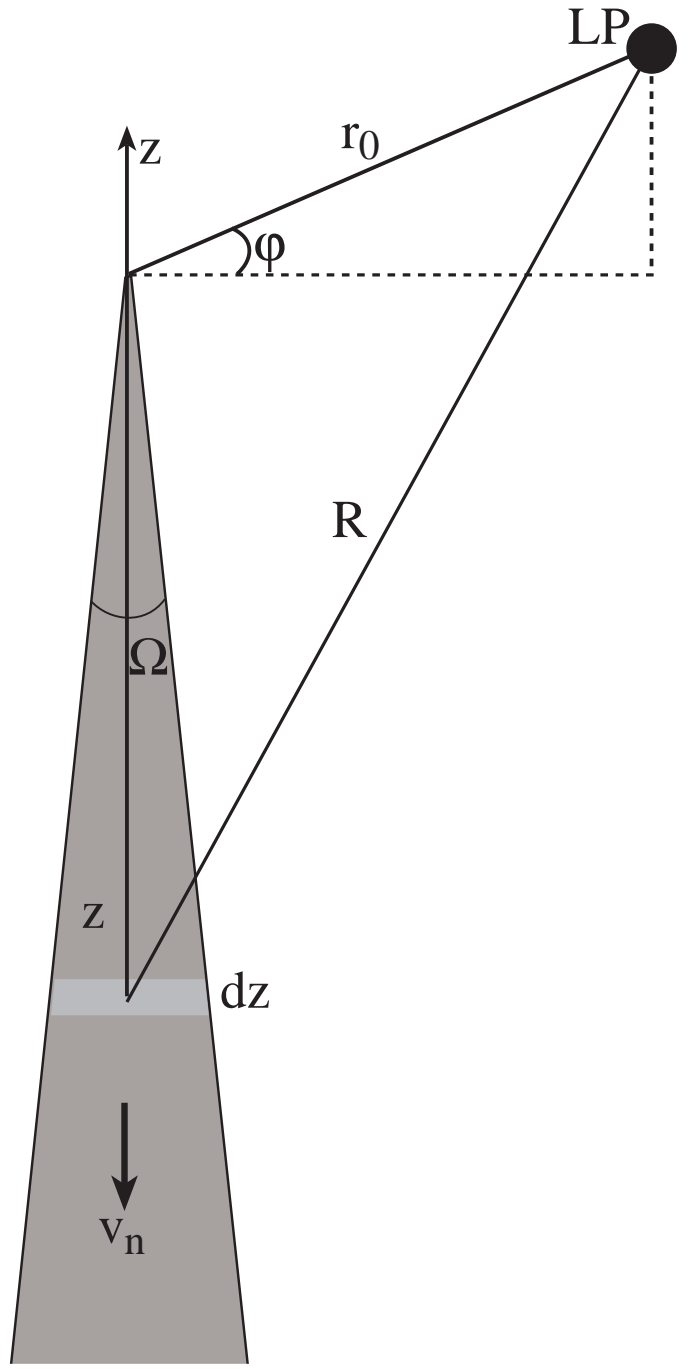

Fig. 7. Schematic representation of the gas release cone; the opening solid angle of the release is $\Omega$. The observation point (L2 sensor) is at distance $r_{0} \approx 8 \mathrm{~m}$ from the source of the release.

current is much larger than the current contributed by the environment. On the other hand, the spacecraft with a conductive surface floats in the ambient medium so that its potential varies inversely with the density $\left(N_{e}\right)$. Therefore, the difference $V_{2 s}$ also changes with $N_{e}$ in a way that depends upon the energy distribution of the photoelectrons escaping from the satellite surface (Laakso and Pedersen, 1998).

Figure 8 displays the electron density $N_{e}$ against the potential difference $-V_{2 s}$ for the time intervals under consideration (Figs. $4 \mathrm{a}$ and $4 \mathrm{~b}$ ), where $N_{e}$ is obtained with the swept Langmuir probe (L1). The solid and dotted curves are shown for comparison; they represent the similar relationships for the Polar satellite (an Earth orbiter). The reference electrodes on Polar are located at distances of $65 \mathrm{~m}$ (sensors 1 and 2) and $6.9 \mathrm{~m}$ (sensors 5 and 6) from the spacecraft body (Harvey et al., 1995). In contrast, the Vega Langmuir probes and electric sensors are located at distances of only 1 and 2 me- 


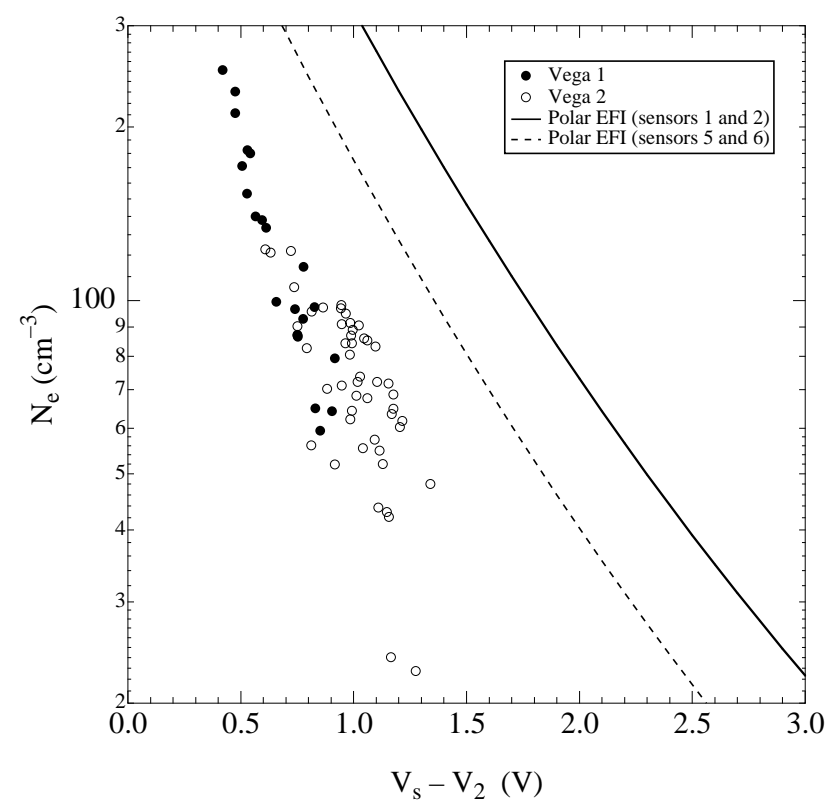

Fig. 8. Potential difference between the spacecraft and the probe plotted against the electron density; full circles are for Vega 1, open squares for Vega 2. The solid and dashed lines show empirical relationships for two different boomlengths of the Polar spacecraft.

ters, respectively, from the solar panels (Fig. 1).

The discrepancies between the various density and voltage characteristics can be ascribed to differences in boom length, plasma environment and solar illumination. The two Vega spacecraft are submitted to a solar flux which is about $50 \%$ larger than at $1 \mathrm{AU}$. Therefore theses spacecraft should assume more positive potentials than Polar in a given plasma environment; this argument, however, does not support the observations and the explanation lies elsewhere, especially since the photoelectron flux plays a minor role in this relationship (for detail, see Laakso and Pedersen, 1998). In fact, the magnitude of the spacecraft potential is underestimated when the sensor is too close to the vehicle; achieving a reasonable accuracy requires that the electrical state of the reference electrode is not influenced by that of the spacecraft. This condition is only fulfilled when the separation between the spacecraft body and the sensor is larger than the Debye length of the ambient plasma.

For a given electron density, the Debye length is shorter in the Vega environment $\left(\lambda_{D} \approx 0.5-1.5 \mathrm{~m}\right)$ than in the Polar environment $\left(\lambda_{D} \approx 1-5 \mathrm{~m}\right)$, since the electron temperature is lower in the cometary and nitrogen plasmas $\left(T_{e} \approx 0.5 \mathrm{eV}\right)$ surrounding Vega than in the Earth's magnetosphere $\left(T_{e} \approx\right.$ $1-100 \mathrm{eV})$; the boomlength is, nevertheless, the parameter which orders the Polar and Vega observations in a logical sequence (Fig. 8).

However, based on Fig. 8, we conclude that the variation of the spacecraft potential in response to the change in ambient electron density can be monitored, even when the reference probe is mounted on a short boom.

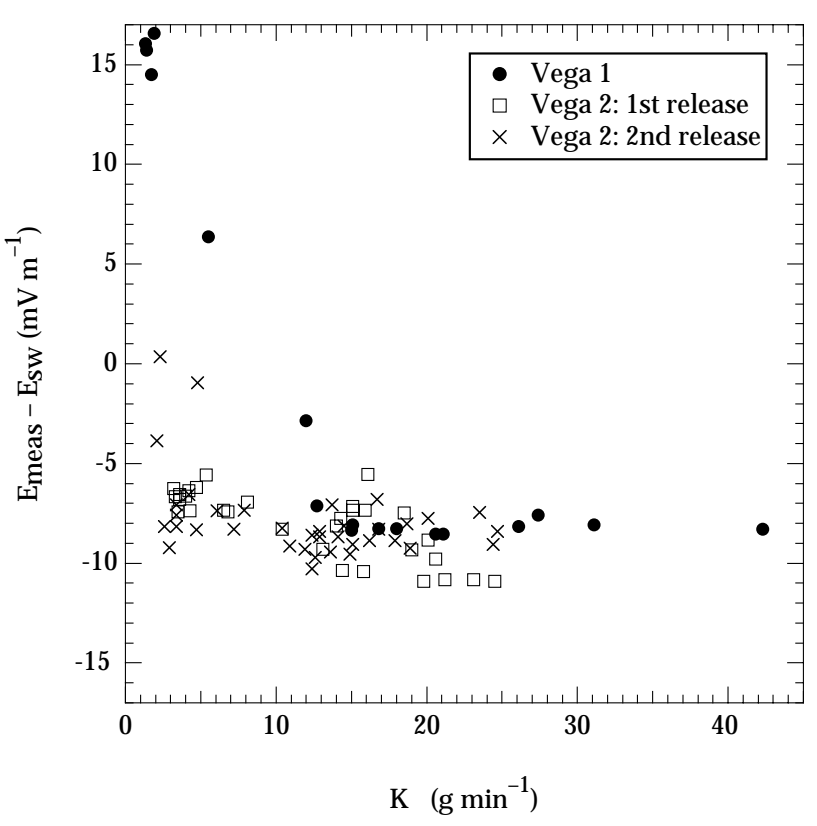

Fig. 9. Quasistatic electric field $E_{y}$ plotted against the gas release rates for Vega 1 and Vega 2.

\subsection{Quasi-static electric field}

The quasi-static electric field, $E_{y}$, is plotted as a function of time in the fourth panel of Figs. 4a and $4 \mathrm{~b}$, and shows significant variations during the gas releases. It is observed that in spite of low telemetry resolution and large data scatter, the electric field levels, initially close to zero (see Figs. 4a and $4 \mathrm{~b})$, settle around negative values of $-15 \mathrm{mV} \mathrm{m}^{-1}$ on Vega $1(K>13 \mathrm{~g} / \mathrm{min})$ and $-4 \mathrm{mV} \mathrm{m}^{-1}$ on Vega $2(K>5$ $\mathrm{g} / \mathrm{min}$ ), before returning to positive levels of $5-10 \mathrm{mV} \mathrm{m}^{-1}$ toward the end of the events.

Measuring electric fields is a complex operation, especially with short booms. The observed signal may result from the superimposition of several phenomena, such as the electric field induced by the solar wind stream, the electron density inhomogeneity between and around the sensors, the spacecraft electrostatic charging, and the polarization of the nitrogen cloud. We shall now assess the relative importance of each contribution.

\subsubsection{Induced electric fields}

The induced electric field is

$E_{s w}=-V_{s w} B_{z} \cos \alpha$

where $V_{s w}$ is the velocity of the solar wind (Gringauz et al., 1986), $B_{z}$ is the component of the magnetic field perpendicular to the ecliptic (Schwingenschuh et al., 1986), and $\alpha$ is the angle between the spacecraft's z-axis and the sunward direction.

The measured electric field $E_{\text {meas }}$ differs from $E_{s w}$ before, during, and after the release (see Table 1). Figure 9 is a plot 
Table 1. The average induced and measured electric fields during the gas releases

\begin{tabular}{llrr}
\hline variable & units & Vega 1 & Vega 2 \\
\hline$V_{s w}$ & $\mathrm{kms}^{-1}$ & 510 & 620 \\
$B_{z}$ & $n T$ & 12 & -10 \\
$\alpha$ & $\circ$ & 18 & 15 \\
$E_{\text {sw }}$ & $m V m^{-1}$ & -6 & 5 \\
$E_{\text {meas }}$ & $m V m^{-1}$ & -15 & -4 \\
\hline
\end{tabular}

of the electric field difference, $E_{a}=E_{\text {meas }}-E_{s w}$, against the release rate $K$ during the release. The difference in $E_{a}$ is about $-8 \pm 1 \mathrm{mV} \mathrm{m}^{-1}$, which reflects the relative consistency of the measurements when the leak rate exceeds $5 \mathrm{~g}$ $\min ^{-1}$. This is due to the fact that a large electron density provides a better environment for quasi-static electric field measurements; since the Debye length is shorter, the antenna impedance is reduced and the asymmetry due to photoemission plays a relatively less important role (for more details about the probe impedance in the low-frequency regime, see Laakso et al., 1995) Thus, the nitrogen release seems to create a favourable plasma environment for electric field measurements. However, an additional explanation is needed to resolve the observed stray field; for example, if the floating potential of sensor $\mathrm{P} 2$ is $0.1 \mathrm{~V}$ above that of $\mathrm{P} 1$, it yields an electric field of $-9 \mathrm{mV} \mathrm{m}^{-1}$.

\subsubsection{Electric fields induced by electron density inhomo-} geneities

A difference of $0.1 \mathrm{~V}$ between the potentials of the electric sensor during the gas releases, corresponding to a spurious electric field $E_{a}=-9 \mathrm{mV} \mathrm{m}^{-1}$, can possibly be explained by a discrepancy in the electron density at the locations of $\mathrm{P} 1$ and P2, due to the fact that the nitrogen source of IKS is closer to P1 than to P2 (see the IKS camera in Fig. 1).

Figure 10 shows Vega 1 electric field and plasma density measurements. The panels from top to bottom are the currents collected by L1 and L2, when both probes are biased at $+5 \mathrm{~V}$ with respect to the spacecraft; electron density determined from L2 measurements; and the dc electric field. A similar comparison cannot be made for Vega 2 because L1 and L2 are never biased at the same potential (for detail about the potential sweep of L1, see Sect. 2). According to the top panel, before and after the release, the electron density is $5-10 \%$ higher at probe L2 (the lagging probe with respect to the velocity of the spacecraft) than at probe L1, whereas the densities are very similar during the release; we may assume that the same situation applies to the densities at probes $\mathrm{P} 1$ and $\mathrm{P} 2$. One may ponder whether electron temperature variations can cause the observed variations in the electron current. It is quite unlikely as the electron temperature plays a minor role in the electron flux collected by an

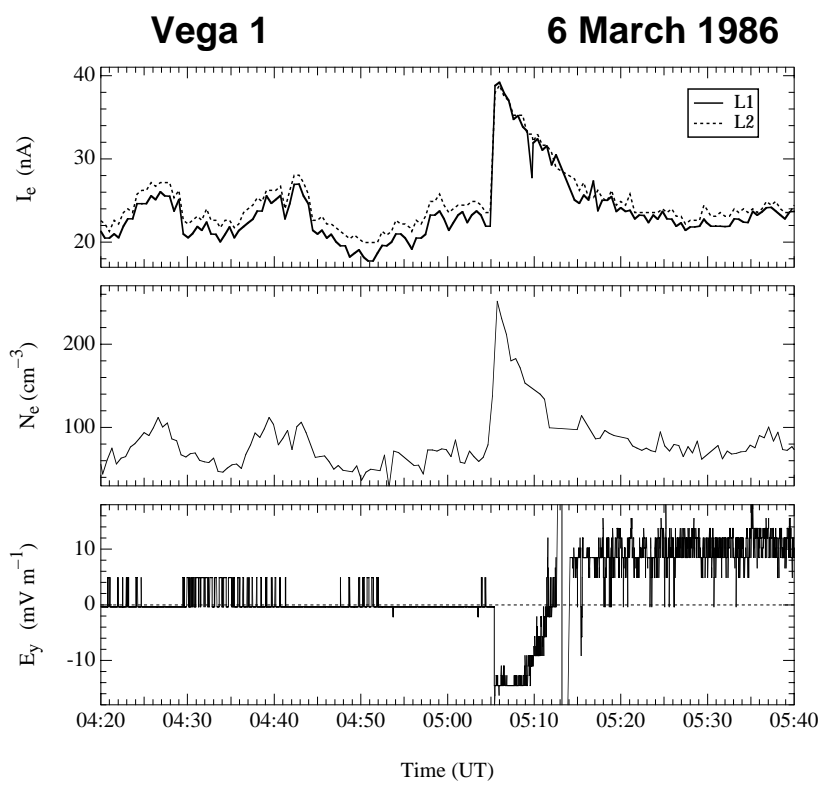

Fig. 10. Plasma and field measurements for Vega 1 at 04:20-05:40 UT. The panels, from top to bottom, are the electron fluxes at L1 and L2, electron density derived from L1 data, and dc electric field.

electric probe in a tenuous environment (for more details, see Laakso and Pedersen, 1998).

Due to a lower density environment, probe P1 develops a more positive potential than probe $\mathrm{P} 2$, which is equivalent to a spurious electric field, $E_{a}>0$, oriented from P1 to P2. Figure 11 shows the predicted magnitudes of $E_{a}$ against the electron density $N_{e}$ for relative density differences of $1 \%$, $5 \%, 10 \%$ or $20 \%$ between the probes, and the electron temperatures $T_{e}$ of $0.5 \mathrm{eV}, 1 \mathrm{eV}$, and $1.5 \mathrm{eV}$ (for more details of the analysis, see Laakso et al., 1995). According to Fig. 10, the relative density difference between $\mathrm{P} 1$ and $\mathrm{P} 2$ is about $10 \%$, with the average density in the range of $50-100 \mathrm{~cm}^{-3}$, and the electron temperature is $0.5-1 \mathrm{eV}$; therefore we expect $E_{a}$ to be about $+7 \mathrm{mV} \mathrm{m}^{-1}$.

Before the gas releases on Vega 1, occur this spurious effect superposed upon a real field of $-5 \mathrm{mV} \mathrm{m}^{-1}$ yields a signal close to zero or somewhat positive that is, in fact, measured by Vega 1. However, during the release, the electron current seems to be very similar at two probes, and the electron density inhomogeneity cannot explain the large electric field appearing during the releases.

\subsubsection{Polarization of the released nitrogen}

The large negative electric fields in Figs. $4 a$ and $4 b$ within the nitrogen cloud are not fully explained by the previous sources. A possible source may be polarization electric fields that have been observed during chemical releases. The characteristic features of some releases are compared in Table 2. In spite of the fact that similar numbers of neutral atoms are injected in all cases, it is improbable that the Vega events lead to observable effects because: 


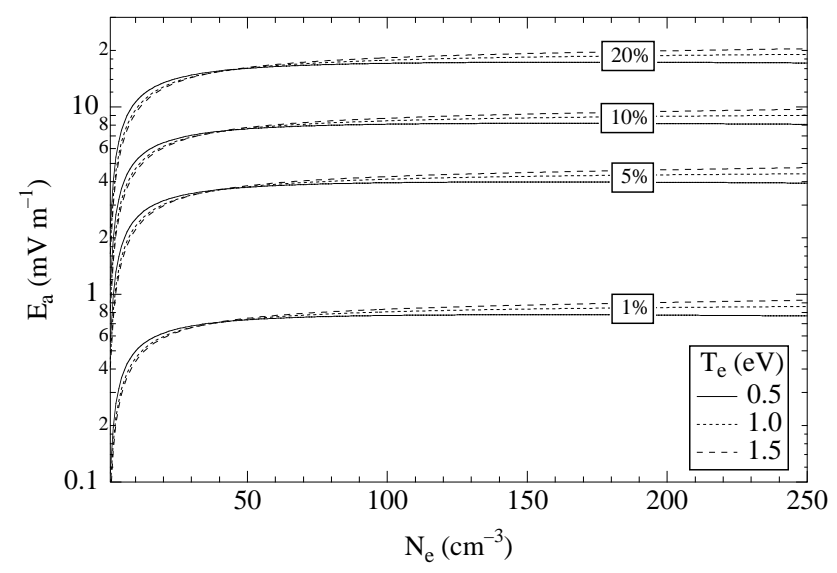

Fig. 11. Levels of spurious electric field which develops when the electron density around P1 differs from that around P2 by a given percentage. The density gradients along the antenna baseline are $1 \%, 5 \%, 10 \%$, and $20 \%$ between 0 and $260 \mathrm{~cm}^{-3}$, and the electron temperature is selected between $0.5 \mathrm{eV}, 1 \mathrm{eV}$, and $1.5 \mathrm{eV}$.

(1) the ionization time of $\mathrm{N}_{2}$ is several orders of magnitude longer than those of $\mathrm{Ba}$ and $\mathrm{Li}$;

(2) the $\mathrm{N}_{2}$ releases are spread out over periods of time longer than the lifetime of clouds generated by explosive charges;

(3) the cloud obviously remains too small with respect to the electron and ion gyroradii that any charge separation could appear;

(4) the $\mathrm{N}_{2}$ beams are collimated by a nozzle and do not form a spherical cloud;

(5) the spacecraft is always located at one end of the beam rather than being immersed in the center of a cloud.

Also note that, in general, if the conductivity is high in the cloud, polarization decreases rather than increases the ambient electric field. However, as pointed out in (4) and (5) above (see our analysis in Sect. 3.1) the spacecraft stays at the edge of the cloud instead of inside of it. In fact, another feature of the polarization of an ionized cloud in a magnetized medium is the appearance of large electric fields outside the cloud (Haerendel et al., 1986). The corresponding electric field is approximately twice as much as the original electric field (Cheng, 1987). This is an appealing explanation for Vega 1 where the electric fields are approximately doubled, but not for Vega 2, where the observed field should point to the opposite direction. Note that on Vega 2, the induced electric field is +6 volts, and then the polarization electric field is expected to be +12 volts, whereas the measured field is -4 volts. Thus, the polarization electric fields cannot be considered as a potential source of the electric fields during the releases.
Table 2. Comparison between the AMPTE and Vega releases

\begin{tabular}{lccc}
\hline \multirow{2}{*}{ Parameter } & \multicolumn{2}{c}{ AMPTE } & Vega \\
& $\mathrm{Ba}$ & $\mathrm{Li}$ & $N_{2}$ \\
\hline atomic mass $(\mathrm{amu}), m_{0}$ & 137 & 7 & 28 \\
mass release $(\mathrm{kg}), M$ & 2 & 2 & 0.7 \\
number of atoms, $N_{0}$ & $10^{25}$ & $5 \cdot 10^{25}$ & $1.5 \cdot 10^{25}$ \\
ionization time $(\mathrm{s}), \alpha^{-1}$ & $30 \mathrm{~s}$ & $3 \cdot 10^{3}$ & $1 \cdot 10^{5}$ \\
expansion speed $\left(\mathrm{km} \mathrm{s}^{-1}\right), v_{n}$ & 1 & 1 & 0.2 \\
\hline
\end{tabular}

\subsubsection{Spacecraft charging}

Another source of interference is the asymmetry of the electrostatic charge distributed over the surface of the spacecraft, especially as the sensors lie only $2 \mathrm{~m}$ away from the solar panels (Fig. 1). An electric field of $9 \mathrm{mV} \mathrm{m}^{-1}$, for example, can be explained by a difference of $0.1 \mathrm{~V}$ between the ambient potentials at the locations of $\mathrm{L} 1$ and $\mathrm{L} 2$, which requires a somewhat larger difference $\Delta V_{f}$ between the floating potentials of the outer solar panels.

A simple orbit-limited theory provides an estimate of the variation in the satellite floating potential, $\Delta V_{f}$, associated with an increment $\Delta N_{e}$ of the electron density (Laakso et al., 1995)

$\Delta V_{f}=\frac{T_{p h}}{e} \frac{e V_{f}+T_{e}}{e V_{f}+T_{e}+T_{p h}} \frac{\Delta N_{e}}{N_{e}}$,

where $V_{f}$ is the floating potential, $T_{p h}$ is the photoelectron temperature, $T_{e}$ is the ambient electron temperature, and $N_{e}$ is the ambient electron density. We find $\Delta V_{f} \approx 0.1 \mathrm{~V}$ for $\Delta N_{e} / N_{e}=0.1$ and $N_{e} \approx 50-250 \mathrm{~cm}^{-3}$.

According to the measurements in Fig. 10, relative spatial variations of ambient density larger than $10 \%$ are not expected near the probes during the releases, which tends to suggest that spacecraft differential charging cannot be a reason for the electric fields. However, since the solar panel structures are conductive and their potentials are determined by the total environment, it is quite possible that the solar panels are charged differently during the release, although we cannot monitor it. Therefore, this source may nevertheless be the most likely to explain the observations, although we cannot fully prove it.

\subsection{AC electric fields}

The signals delivered by the filter bank connected to the electric antenna and the power integrated in the whole frequency range are displayed in the first and second panels of Figs. 4a and $4 \mathrm{~b}$. The average spectral densities corresponding to the early phase of the nitrogen release on Vega 1 (05:05:30 05:06:30 UT) and Vega 2 (04:34:00-04:34:30 UT) are plotted in Fig. 12. The average spectrum taken before the event on Vega 1, between 04:25:00-04:35:00 UT, is also given for reference; the corresponding background spectrum for 
Vega 2 is not shown, but is similar. Figure 12 also displays, for comparison, four spectra recorded with AMPTE: in the upstream, in the compression region, at the cavity boundary and in the cavity (Gurnett et al., 1986b). AMPTE detected a broadband peak in the solar wind between 2 and $30 \mathrm{kHz}$, below the electron plasma frequency $f_{p e}$ (Gurnett et al., 1986b), similar to that continuously observed on Vega, where $f_{p e}$ is in the range of $30-140 \mathrm{kHz}$ during the releases and of the order of $30 \mathrm{kHz}$ before the releases.

The plasma wave emission increases at all measured frequencies, although the largest enhancement occurs below a few hundred $\mathrm{Hz}$, close to the electron gyrofrequency $(\sim 500 \mathrm{~Hz})$. Due to a lower nitrogen injection rate on Vega 2 the wave disturbances are not as intense as on Vega 1, but the shapes of the spectra are similar. Magnetic wave fields are not measured on Vega, and hence, one cannot be sure whether the disturbances are electrostatic or electromagnetic.

The magnetic field of the waves could not be measured on Vega, but the similarity with the data collected by AMPTE in the solar wind and the compression region suggests that the Vega results are characteristic of a broadband electrostatic noise caused by an ion beam plasma instability, as observed during the AMPTE releases in the compression and upstream regions (Gurnett et al., 1986b). Contrary to AMPTE, the wave intensity is never less than that observed in the upstream, and this confirms that Vega has never entered any cavity.

A puzzling feature is the continuation of significant plasma wave emissions after the release. We found in Sect. 3.1.2 that the nitrogen beam is well collimated, and that one may expect no disturbances after the release. However, it is quite likely that some nitrogen will accumulate on the surface during the releases (lasting for a few tens of minutes), and the disturbances are then due to the desorption of nitrogen from the spacecraft surface. Note that both dc electric fields and electron current observations were also somewhat disturbed after the releases, which may also be caused by the same desorption of nitrogen accumulation. A similar type of observation was made at an Apollo site where large enhancements of the neutral density were detected during the lunar daytime, immediately after sunrise for more than 300 Earth days following the Apollo mission, which is in contradiction with the expected behaviour of the lunar exosphere (Vaniman et al., 1991). The source for this behaviour was the accumulation of exhaust gases on the Apollo site at nighttime and desorption of these gases at daytime.

\subsection{Diamagnetic effects}

No magnetic field perturbations occur during the Vega releases, which suggests that no diamagnetic cavity develops. This hypothesis is supported further by the fact that the wave activity increases during the releases, whereas it should cease in the diamagnetic cavity (Gurnett et al., 1986b; Koons and Anderson, 1988).

Due to the low photoionization rate $\alpha$ of $\mathrm{N}_{2}$ molecules, the plasma density is not high enough to sustain any diamagnetic

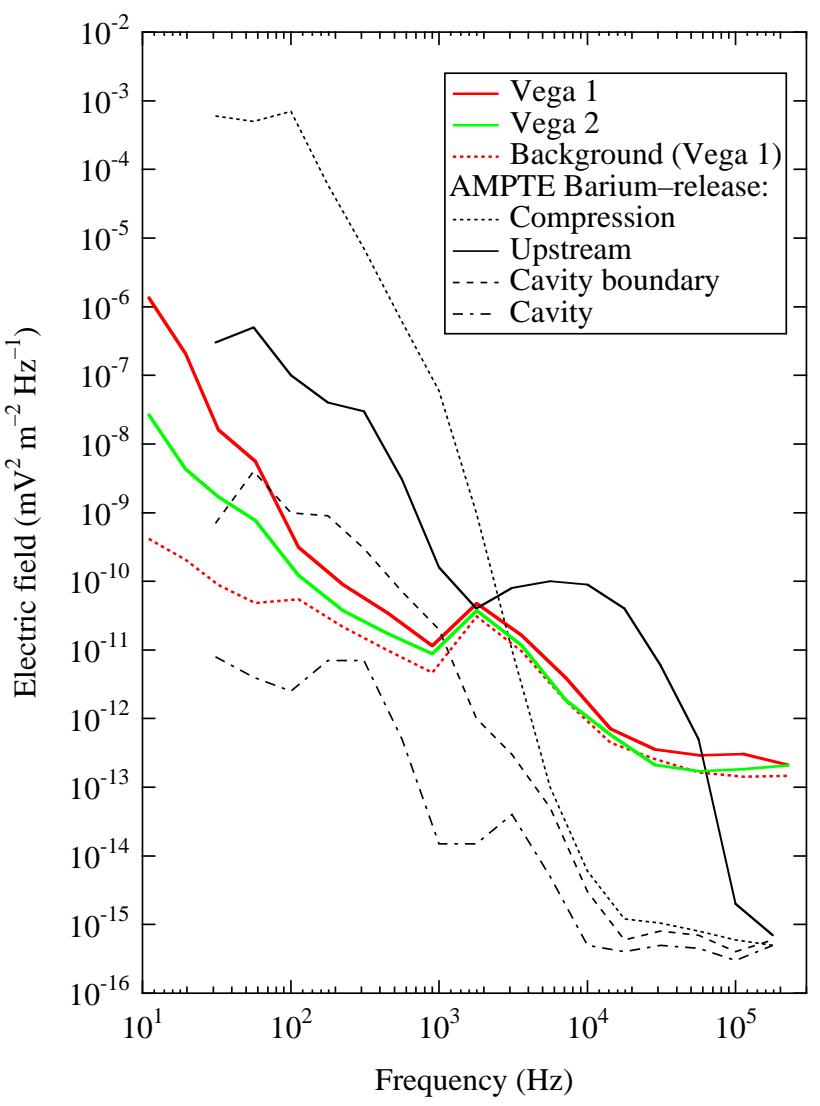

Fig. 12. Plasma wave spectra taken during the IKS gas releases on the Vega spacecraft compared to the AMPTE results reported by Gurnett et al. (1986).

effect. The maximum electron density is only $250 \mathrm{~cm}^{-3}$ during the Vega events, but several $1000 \mathrm{~cm}^{-3}$ in the case of the AMPTE releases (Table 1). The maximum diamagnetic effect should be observed at a time

$T_{0}=\sqrt{\frac{m_{i} N_{0} \alpha}{4 \rho_{s w} v_{s w}^{2} v_{n}}} \sim 0.3 s$

after the release (Haerendel, 1983), where $m_{i}$ is the mass of an $N_{2}^{+}$ion, $N_{0}=N_{A} M / m_{0}$ is the total number of neutrals, $M$ is the total mass of gas, $\rho_{s w}$ is the solar wind mass density (number density $\sim 30 \mathrm{~cm}^{-3}$ ), and $v_{s w} \sim 500 \mathrm{~km} \mathrm{~s}^{-1}$ is the solar wind speed. Note that the numerical value of $T_{0}$ is an upper limit since the neutrals are not released instantaneously.

The maximum ion dynamic pressure at time $T_{0}$ is approximately

$P_{0}=\frac{m_{i} n_{i} v_{n}^{2}}{\frac{4 \pi}{3}\left(v_{n} T_{0}\right)^{3}}=\frac{3 m_{i} \alpha Q}{4 \pi T_{0}^{3} v_{n}}<2 \cdot 10^{-12} P a$,

where $n_{i}$ is the $N_{2}^{+}$density. This pressure is only a small fraction of the solar wind pressure $\left(\sim 10^{-10} \mathrm{~Pa}\right)$. It is thus obvious that the $\mathrm{N}_{2}^{+}$cloud cannot perturb the solar wind stream and, in particular, it cannot keep the solar wind magnetic field out of the plasma cloud. 
Remember, however, that electron density measurements yield that the spacecraft is located outside the release, and thus, the density in the cloud is likely to be much higher. Thus, we cannot say for certain whether the cloud is associated with a shock or even a cavity.

\section{Summary}

The nitrogen gas which is released for the cooling of an infrared spectrometer on the Vega 1 and 2 spacecraft, produces strong plasma and wave disturbances. The maximum release rate is 42 grams per minute, equivalent to $1.5 \cdot 10^{22}$ molecules per second. Neutrals are ionized by solar EUV radiation at a rate of $7 \cdot 10^{-7} \mathrm{~s}^{-1}$, producing a plasma cloud which expands around the spacecraft and interacts with the spacecraft and the solar wind in various ways. The major findings of this investigation are as follows:

(1) Within a few meters from the release point, the electron flux varies linearly with the release rate, $K$, following the relation $\Delta I_{e}=0.33 \cdot K$, where $\Delta I_{e}$ is expressed in $\mathrm{nA}$ and $K$ is expressed in grams per minute.

(2) The comparison between the observations and the model confirms that the gas does not expand spherically but is collimated by the nozzle at least for the first few meters. The observations can be explained by assuming that only $1 \%$ of the ions escape from the beam, which suggests that the spacecraft is not immersed in the cloud, but stays at the edge of an extending filamentary structure.

(3) The potential difference between a biased probe and the spacecraft structure, $V_{2 s}=V_{2}-V_{s}$, is primarily controlled by the plasma density. This study also shows that the spacecraft potential variations can be monitored with a sensor mounted on a relatively short boom.

(4) The gas release increases the plasma density around the spacecraft and improves the consistency of quasistatic electric field measurements. Additional effects are caused by the inhomogeneity of the nitrogen plasma environment.

(5) The plasma wave emission increases by more than two orders of magnitude, primarily below the electron gyrofrequency. The Vega electric field spectra are quite similar to those recorded upstream of the AMPTE ion clouds and are likely to be reminiscent of electrostatic waves generated via an ion beam-plasma interaction, as studied by Gurnett et al. (1986b).

(6) Plasma waves continue for more than one hour after the end of the releases, suggesting that part of the nitrogen gas is first adsorbed on the spacecraft surface and then slowly desorbed.

(7) No diamagnetic cavity is detected during the releases since the ambient density of the $\mathrm{N}_{2}^{+}$cloud remains too low. This view is supported by the existence of a strong plasma wave activity, large quasi-static electric fields and undisturbed interplanetary magnetic fields. However, the spacecraft lies outside the filamentary cloud during the events, and the development of a downstream cavity cannot be excluded with absolute certainty.

Acknowledgements. We are grateful to M. Combes (CNRS-LPSP, Verrieres, France) for providing the data of the IKS nitrogen releases. Thanks are also due to N. Khavenson and A. Skalsky (IKI, Moscow) providing inputs about the attitude of the spacecraft during their encounters with comet Halley.

\section{References}

Arduini, M., Bibring, J. P., Cazes, S., Combes, M., Coron, N., Crifo, J. F., Encrenaz, T., Gispert, R., Harduin, D., Lamarre, J. M., and Malaise, D.: The comet Halley flyby I. R. sounder I. K. S., Adv. Space Res., 2 (4), 113-122, 1983.

Banks, P.M. and Kockarts, G.: Aeronomy, Part A, Academic Press, New York, 1973.

Burke, W. R.: (Ed) Active Experiments in Space, ESA SP-195, ESA, Noordwijk, 1983.

Cheng, A. F.: Tranverse deflection and dissipation of small plasma beams and clouds in magnetized media, J. Geophys. Res., 92, 55-63, 1987.

Grard, R., Laakso, H., Pedersen, A., Trotignon, J. G., and Mikhailov, Y.: Observations of the plasma environment of comet Halley during the Vega flybys, Ann. Geophysicae, 7, 141-150, 1989.

Gringauz, K. I., Gombosi, T. I. , Remizov, A. P., Apáthy, I., Szemerey, I., Verigin, M. I., Denchikova, L. I., Dyachkov, A. V., Keppler, E., Klimenko, I. N., Richter, A. K., Somogyi, A. J., Szegö, K., Szendrö, S., Tátrallyay, M., Varga, A., and Vladimirova, G. A.: First in situ plasma and neutral gas measurements at comet Halley, Nature, 321, 282-285, 1986.

Gurnett, D. A., Anderson, R. R., Ma, T. Z., Haerendel, G., Paschmann, G., Bauer, O. H., Treumann, R. A., Koons, H. C., Holzworth, R. H., and Lühr, H.: Waves and electric fields associated with the first AMPTE artificial release, J. Geophys. Res., 91, 10013-10 028, 1986b.

Haerendel, G.: The role of momentum transfer to the ambient plasma in critical-velocity experiments, in: Active Experiments in Space, ESA SP-195, 337-340, ESA/ESTEC, Noordwijk, 1983.

Haerendel, G., Bauer, O. H., Cakir, S., Höppl, H., Rieger, E., and Valenzuela, A.: Coloured bubbles - an experiment for triggering equatorial spread F, in: Active Experiments in Space, ESA SP195, 295-298, ESA/ESTEC, Noordwijk, 1983.

Haerendel, G., Paschmann, G., Baumjohann, W., and Carson, C. W.: Dynamics of the AMPTE artificial comet, Nature, 320, 720723, 1986.

Harvey, P., Mozer, F. S., Pankow, D., Wygant, J., Maynard, N. C., Singer, H., Sullivan, W., Anderson, P. B., Pfaff, R., Aggson, T., Pedersen, A., Fälthammar, C.-G., and Tanskanen, P.: The electric field instrument on the Polar satellite, Space Sci. Rev., 71, 583596, 1995.

Holmgren, G., Boström, R., Kelley, M. C., Kintner, P. M., Lundin, R., Fahleson, U. V., Bering, E. A., and Sheldon, W. R.: Trigger, an active release experiment that stimulated auroral particle pre- 
cipitation and wave emissions, J. Geophys. Res., 85, 5043-5053, 1980.

Koons, H. C. and Anderson, R. R.: A comparison of the plasma wave spectra for the eight AMPTE chemical releases, J. Geophys. Res., 93, 10 016-10 024, 1988.

Laakso, H.: Electric fields and cold electrons in the vicinity of comet Halley, J. Geophys. Res., 96, 7731-7757, 1990.

Laakso, H. and Pedersen, A.: Ambient electron density derived from differential potential measurements, in: Measurement Techniques in Space Plasmas, (Eds) Pfaff, R. F., Borovsky, J. E., and Young, D. T., AGU Monogram 102, 49-54, AGU, Washington, D. C., 1998.

Laakso, H., Aggson, T., and Pfaff, R.: Plasma gradient effects on double probe measurements in the magnetosphere, Ann. Geophysicae, 13, 130-146, 1995.

Lühr, H., Southwood, D. J., Klöcker, N., Acuña, M., Häusler, B., Dunlop, M. W., Mier-Jedrzejowicz, W. A. C., Rijnbeek, R. P., and Six, M.: In situ magnetic field measurements during AMPTE solar wind $\mathrm{Li}^{+}$releases, J. Geophys. Res., 91, 1261-1270, 1986.

Sasaki, S.: Results from gas injection experiment in SEPAC, J. Geomagn. Geoelectr., 40, 1193-1204, 1988.

Schwingenschuh, K., Riedler, W., Schelch, G., Yeroshenko, Y. G., Styashkin, V. A., Luhmann, J. G., Russell, C. T., and Fedder, J. A.: Cometary boundaries: Vega observations at Halley, Adv. Space Res., 6 (1), 217-228, 1986.

Valenzuela, A., Haerendel, G., Höppl, H., Melzner, F., Neuss, H., Rieger, E., Stöcker, J., Bauer, O., Höfner, H., and Loidl, J.: The AMPTE artificial comet experiments, Nature, 320, 700-703, 1986.

Vaniman, D., Reedy, R., Heiken, G., Olhoeft, G., and Mendell, W.: The lunar environment, in: Lunar Sourcebook - A User's guide to the Moon, (Eds) Heiken, G. H., Vaniman, D. T., and French, B. M., 27-60, Cambridge Univ., 1991. 